\title{
The role of innate lymphoid cells in selected disease states - cancer formation, metabolic disorder and inflammation
}

\author{
Agata Poniewierska-Baran ${ }^{1}$, Beata Tokarz-Deptuła' ${ }^{1}$, Wiesław Deptuła²
}

\begin{abstract}
${ }^{1}$ Department of Immunology, Faculty of Biology, University of Szczecin, Szczecin, Poland

${ }^{2}$ Veterinary Center of the Nicolaus Copernicus University, Torun, Poland
\end{abstract}

Submitted: 23 June 2017

Accepted: 13 October 2017

Arch Med Sci 2021; 17 (1): 196-206

DOI: https://doi.org/10.5114/aoms.2019.89835

Copyright @ 2019 Termedia \& Banach

\section{Abstract}

Innate lymphoid cells (ILCS) are a recently described group of immune cells that can regulate homeostasis and protect mammalian organisms, including humans, from infections and diseases. Considering this, ILC research is still ongoing to better understand the biology of these cells and their roles in the human body. ILCs are a multifunctional group of immune cells, making it important for the medical community to be familiar with the latest research about the ILC families and their functions in selected disease states, such as cancer formation, metabolic disorders and inflammation. By discovering the roles of ILC populations and their participation in many disorders, we can improve disease diagnostics and patient healthcare.

Key words: innate lymphoid cells, innate immunity, natural immunity cells.

\section{Families of innate lymphoid cells}

Innate lymphoid cells (ILCS) were recently described as an element of natural immunity that can take part in maintaining homeostasis in organisms and participate in infections and infectious diseases [1-24]. Innate lymphoid cells can appear in digestive, respiratory and urogenital systems, as well as in skin, adipose tissue, blood and many internal organs [24-27]. Adamiak et al. [28] characterized the ILC families as: NK (natural killer) cells producing mainly interferon $\gamma($ IFN- $\gamma$ ), lymphoid tissue inducer (LTi) cells, ILC22 cells with similar features as NK and LTi cells, ILC17 cells producing interleukin 17 (IL-17), ILC2 cells which are natural T helper 2 (nTH2) cells, nuocytes, innate helper type $2(\mathrm{IH} 2)$ cells and MPP type 2 cells (multifocal progenitor type 2). Another known and widely used classification of ILCS is based on their origin [29] and their ability to produce cytokines and key transcription regulators [30]. This classification, also presented in Table I, distinguishes three groups of cells. Group 1 (ILC-1) comprises IL-12, IL-15 and IL-18-responsive cells, that can activate transcription factor T-bet and can secrete IFN- $\gamma$ or tumor necrosis factor (TNF) $[23,31]$. Group 2 (ILC-2) is formed by IL-25, IL-33 and thymic stromal lymphopoietin (TSLP)-sensitive cells, which can activate the GATA-3 transcription factor (trans-acting-cell-specific transcription factor), and can produce IL-5, IL-13, IL-4 and amphiregulin (Areg) - a pro-

\author{
Corresponding author: \\ Beata Tokarz-Deptuła \\ Department of Immunology \\ Faculty of Biology \\ University of Szczecin \\ 3C Felczaka St \\ 71-412 Szczecin, Poland \\ Phone: +48914441610 \\ Fax: +48 914441606 \\ E-mail: \\ kurp13@univ.szczecin.pl
}


Table I. Scheme of innate lymphoid cell division into three groups taking into account the production of cytokines and their potential effects $[23,102]$

\begin{tabular}{|cccc|}
\hline Name & Stimulation & Secretion & Function \\
\hline Group 1 (ILC-1): & & & \\
\hline NK & IL-12, IL-15, IL-18 & IFN- $\gamma$, TNF- $\alpha$, perforins, granzymes & Antiviral, antitumor \\
\hline ILC1 & IL-12, IL-15, IL-18 & IFN- $\gamma$, TNF- $\alpha$ & Antibacterial \\
\hline Group 2 (ILC-2) & IL-25, IL-33, TSLP & $\begin{array}{c}\text { IL-5, IL-4, IL-6, IL-9, IL-13 } \\
\text { amphiregulin }\end{array}$ & $\begin{array}{c}\text { Antiparasitic, antiallergic, } \\
\text { cell metabolism regulators }\end{array}$ \\
\hline Group 3 (ILC-3) & IL-1 $\beta$, IL-23 & IL-17a, IL-22, GM-CSF, IFN- $\gamma$ & $\begin{array}{c}\text { Antibacterial, antifungal, lymphoid } \\
\text { tissue development regulators }\end{array}$ \\
\hline
\end{tabular}

tein which belongs to the epidermal growth factors [23]. Group 3 (ILC-3) is formed by IL-1 $\beta$ and IL-23-responsive cells, which activate the ROR $\gamma \mathrm{t}$ transcription factor and secrete IL-17a, IL-22, granulocyte-macrophage colony-stimulating factor (GM-CSF) and IFN- $\gamma$ [31]. According to available data, there are few methods for determination ILC cells, partly because of technical limitations, such as flow cytometry [32], mass cytometry [33] and confocal microscopy [34]. Unfortunately, most of the authors used their own strategies for gating ILCS, depending on the tissue being examined, or experimental model (mouse/human), which means they used a specific panel of antibodies for determination of each organ-specific group of ILC. For example, the Bal et al. [35] gating strategy by flow cytometry defined ILCS as negative for lineage markers ( $\mathrm{Lin}^{-}$: CD1a-CD3-CD 14-CD16-CD19-CD34-CD94-CD123BDCA2 ${ }^{-}$FCeR $\left.1 \alpha^{-} \mathrm{TCR} \alpha \beta^{-} \mathrm{TCR} \gamma \delta^{-}\right)$and $\mathrm{CD} 45^{+} \mathrm{CD} 127^{+}$ CD161+. Silver et al. [36] identified ILCs in the peripheral blood with the gating strategy as CD45 Viable $\mathrm{CD}^{-}{ }^{-} \mathrm{CD} 19^{-} \mathrm{Lin}^{-} \mathrm{IL}-7 \mathrm{R} \alpha^{+} \mathrm{CD} 56^{-}$. The most notable and quotable article describing the populations of ILCS and their quantities in various human tissues was published in 2017 , by Simoni et al. [33]. They profile human ILCs using one method, mass cytometry, and using one gating strategy for each ILC group, across tissues. This data give valuable and limited information about differences in the number of ILC cells, depending on tissues and normal/abnormal conditions. Human tissues can be divided into two categories based on their overall ILC subset composition. Under non-pathological conditions, lung and non-mucosal tissues such as peripheral blood, cord blood, bone marrow and spleen displayed low frequency of ILC-2 and ILC- 3 cells (less than $5 \%$ of ILC), this questioning the importance of these cells in tissue homeostasis and immune surveillance in comparison to NK cells (more than 95\%). In these tissues, NKp44+ ILC-3 cells represented less than $0.5 \%$ of all ILCs. This observation does not undermine the role of these cells in infections and pathologies such as lung inflammation, where it is clear that rare cells can have profound effects [33]. Importantly, the expression of various markers on ILCs can be modulated in response to cytokine exposure (e.g. CD127, CD94, CRTH2), so the gating strategy should be adjusted depending on the tissue and/ or inflammatory conditions.

By characterizing ILC cells within these three groups (Table I), it should be noted that the ILC-1 group is composed of NK and ILC1 cells, which are mainly involved in the immunity against intracellular bacteria, viruses and tumor cells [37-40]. NK cells exhibit cytotoxic capabilities similar to $\mathrm{CD}^{+}$ T cells [41], whereas ILC1 cells, by restricted cytotoxicity, are more closely related to Th1 (T helper) cells, which can activate mononuclear $(\mathrm{MN})$ and polymorphonuclear (PMN) cells by increasing their capacities for cytotoxicity, phagocytosis, and neutrophil extracellular trap (NET) formation [38, 39, 42]. Group 1 ILCs include NK cells with phenotype CD ${ }^{-}{ }^{-}{ }^{-} 94^{+}{ }^{+}$CD $56^{+}$cells [43] and ILC1 cells which are $\mathrm{Lin}^{-} \mathrm{CD} 127^{+} \mathrm{C}-\mathrm{kit}^{-} \mathrm{CRTH} 2^{-}$[44]. Simoni et al. [33] described the ILC-1 group of NK and ILC1 cells, by $\alpha \mathrm{CD} 14, \alpha \mathrm{CD} 3, \alpha \mathrm{CD} 19, \alpha \mathrm{CD} 56, \alpha \mathrm{CD} 103$. NK cells, as well as ILC1, after IL-12, IL-15 or IL-18 stimulation, can increase secretion of TNF - which is cytotoxic to multiple tumor cell lines and bacteriaand virus-infected cells, but also increases synthesis of IFN- $\gamma$ - which activates MN cells, including macrophages and also NK cells [37-40]. The rapid induction of IFN- $\gamma$ by innate cytokines, such as IL-12 and IL-18, is critical for immunity against infectious pathogens. The combination of IL-12 and IL-18 induces IFN- $\gamma$ expression not only in NK cells but also in B, T and dendritic cells (DCs) [45]. Wang et al. [46] found that a lymphocyte subpopulation of NK-like B cells existed in spleen and mesenteric lymph nodes, had unique features that differed from $T$ and $B$ cells, and can produce IL-12 and IL-18 at an early phase of infection. These NK-like $B$ cells played a critical role in eradication of microbial infection via secretion of IL-12 and IL-18 [46]. Simoni et al. [33] was not able to detect ILC1 cells in any of the tissues assessed, but they identified intra-epithelial-ILC1-like cells, that do not express CD127, but are capable of producing 
cytotoxic granules. These cells represent a broader category of NK cells in mucosal and non-mucosal pathological tissues [33]. It is plausible that contamination with T cells, DCs, ILC3 cells, HSC and NK cells might have resulted in an inaccurate definition of ILC1 cells in their previous studies. Compared to NK cells from the same tissue, these intra-epithelial-ILC1-like cells expressed CD103, the transcription factor T-bet, as well as surface NK cell markers CD56, NKp46, CD94, 2B4, CD161, CD160, CD122, CD69 and CD49a, but did not express CD16 or CD127. According to Simoni et al., these intraepithelial-ILC1-like cells were found in non-pathological tissues only in tonsils (5\%) and colon tissue (25\%) [33].

ILC-2 cells exhibit a protective effect of innate immune response against parasites, but they can also control cell metabolism, and participate in allergic reactions and wound healing [47-60]. In mice, ILC-2 cells are typically identified by flow cytometry and the expression of CD25, KLRG1, ICOS, or ST2 (subunits of the IL-33 receptor complex), but in humans by CD161, ST2, CRTH2 expression [49], or by $\mathrm{Lin}^{-} \mathrm{CD} 127^{+} \mathrm{C}-\mathrm{kit}^{+} \mathrm{CRTH} 2^{+} \mathrm{NCR}^{-}$ phenotype [44]. For ILC subsets, Simoni et al. [33] reported that samples were depleted of contaminating cells by using $\alpha \mathrm{CD} 3, \alpha \mathrm{CD} 20, \alpha \mathrm{CD} 8, \alpha \mathrm{CD} 4$, $\alpha C D 34, \alpha C D 16$ and anti-Mouse IgG microbeads (Miltenyi). Then, cells were stained with an antibody cocktail containing $\alpha$ CRTH2-FITC, $\alpha$ CD127-biotinylate and $\alpha$ NKp44-APC. After washing, cells were stained with $\alpha$ CD94-PercP-Cy5.5, ac-KITBV421, Streptavidin PE-Cy7, and lineage markers $\alpha C D 14-P E, \alpha C D 5-P E, \alpha C D 11 C-P E, \alpha C D 19-P E$, $\alpha$ FCER1 $\alpha-P E, \quad \alpha C D 123-P E$ [33]. Cytokines such as IL-25, IL-33 and thymic stromal lymphopoietin (TSLP) stimulate ILC-2 to secrete IL-5 and IL-13 [47-52, 61], although some of them can also produce IL-4, IL-6, IL-9 and amphiregulin [53-55, 62-64]. By using mass cytometry, Simoni et al. found that ILC-2 cells produced IL-4, IL-5, IL-9, IL-13, GM-CSF, IL-6 and IL-8 in response to IL-18 stimulation [33]. The key transcription factor for ILC- 2 cells and for Th2 lymphocytes is GATA-3 [53, 65]. The ILC-2 cells appear in the spleen (1\%), lung (2\%), colon (3\%), adenoid (7\%), tonsil (13\%) and mucous membranes of the nasal cavity, intestines, as well as skin (40\%), and are thus involved in chronic allergic skin disorders $[33,57,66,67]$ and paranasal sinuses $[49,51,59,60]$. In human skin, ILC- 2 cells represented the main population of ILCS (40\%) and the frequency of ILC-2 cells is higher in cord blood ( $2.7 \%$ cells) as compared to adult blood ( $0.63 \%$ cells) [33]. Their study demonstrates that ILC- 2 cells in cord blood are functionally similar to ILC- 2 cells derived from adult blood after stimulation with IL-33. In pathological tissues, ILC-2 and ILC-3 cells are recruited in tissues, e.g. in colorec- tal tumors and lung tumors, but more than 95\% of ILCs infiltrating these inflamed tissues were composed of NK and ILC1-like cells [33].

The third group from the ILC family is a very heterogeneous group of ILC-3 cells that contains LTi cells with their expression of $\mathrm{CCR}^{+}$, cells with the expression of $\mathrm{NKp} 46^{+}$, and double negative

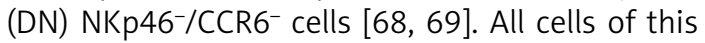
group participate in immunological resistance against bacterial and fungal infections and participate in the development and repair of lymphoid tissue [68-77]. The flow cytometry analyses of the ILC-3 group include $\mathrm{Lin}^{-} \mathrm{CD} 127^{+} \mathrm{C}-\mathrm{kit}^{+} \mathrm{CRTH} 2^{-}$ NKp46 $/$ NKp44 $^{+}$marker expression [44]. Hazenberg Spits [32] demonstrated that a clear definition of ILC-2 and ILC-3 populations in humans by multi-color flow cytometry requires at least eight fluorescence channels. Consequently, technical limitations of flow cytometry devices such as spectral overlap hamper a further in-depth characterization of these cells. Activated ILC-3 cells can synthesize IL-17A, IL-22, TNF, GM-CSF and IFN $\gamma$ [68-72, 78-80]. By using mass cytometry, Simoni et al. [33] found that ILC-3 cells can also produce IL-8 in response to stimulation with IL-18, as well as in combination with IL-23. They did not detect production of IL-22 in response to IL-18 stimulation, but they observed IFN secretion in the presence of IL-18 and IL-23. This results confirms that ILC -3 cells can be plastic in terms of cytokine production in vitro [33]. ILC-3 also features the CCR6 ${ }^{+}$ cell population - which includes the $\mathrm{CD}^{+} \mathrm{T}$ cells and $\mathrm{CD}^{-} \mathrm{T}$ cells and the $\mathrm{CCR}^{-}$cell population which expresses NKp46 in mice, and NKp44 in humans [13]. The frequency of NKp44--ILC3 cells is higher in human cord blood (3.09\% cells) as compared to adult blood (1.05\% cells) [33] and they also occur in blood marrow (2\%), spleen (3\%), skin (18\%), lung (2\%), tonsil (30\%), adenoid (17\%) and colon (15\%) [33], but NKp44+-ILC-3 cells are present mostly in mucosal tissues, such as tonsil (18\%), adenoid (49\%), and colon (23\%) [33].

\section{Innate lymphoid cells and cancer formation}

Interactions between cells of the immune system (IS), including ILCS, and tumor-developing cells with the cells that make the tumor microenvironment, play a key role in cancer formation and metastasis. The connection between tumor cells and IS cells may occur via cytokines, adhesion molecules, which may be secreted either by the host cells or by the cells of the developing tumor, or by means of the so-called microbubbles and metalloproteinases that can help spread the cancer [81]. The first response to tumor formation is the mobilization of IS cells, which activates cytotoxic mechanisms via the production of cytokines (including antitumor ones) to induce apoptosis, resulting in 
the death of cancer cells [82]. When proliferating cancer cells gain an edge over immune system cells, cancer cells take over the control of all microenvironment cells and begin to produce pro-tumor growth factors [81-83]. Among tumor-forming microenvironment cells we can distinguished T lymphocytes, including $\mathrm{Th}_{1}, \mathrm{Th}_{2}$, Treg and $\mathrm{Th}_{17}$, B lymphocytes, NK cells, natural killer T (NKT) cells, DCs, macrophages (M1 and M2), and myeloid derived suppressor cells (MDSC), as well as the families of ILCs - groups 1,2 and 3 [84, 85]. Innate lymphoid cells, which are a source of cytokines, can be involved in the progression of early stages of the disease as well as tissue repair during damage. Therefore, ILC families play an important role in immune activation and the restoration of tissue continuity [86]. This particular ability of ILC cells suggests that they may be involved in the formation and development of tumors and may influence the microenvironment around developing tumor cells. In order to initiate an immune response in a damaged, but non-infected tissue, a number of elements need to cooperate, including damage-associated molecular pattern (DAMP), DCs and macrophages, as well as epithelial cells with active inflammasomes. Inflammasomes are intracellular proteinaceous complexes, whose function is to activate a proinflammatory cascade that leads to pro-caspase-1 synthesis, and activate the family of ILC cells, by IL-18 (ILC-1), IL-33 (ILC-2), and by IL-1 $\beta$ (ILC-3) [87]. High activity of ILC cells can also induce tumor growth [88].

ILC-1 cells have the ability to reduce tumor development by cytotoxic activity and secretion of IL-2, IL-12, IL-18 and IL-21 [89]. In contrast, during tumor development, NK cells belonging to the ILC-1 group can lose the expression of receptors responsible for antitumor activity, such as CD16, CD69, and CD161, and enhance the expression of receptors, e.g. CD158, which can inhibit antitumor receptors [89]. This condition leads to the imbalance of homeostasis and induces immunosuppression, which is also accompanied by a decreased number of NK cells in the blood and, as a result, further development of the cancer [89]. However, DCs present in the tumor microenvironment affect NK cells resulting in positive feedback, as DCs specializing in antigen presentation and activation of the immune response can support cytokine production by NK cells and enhance their cytotoxicity to tumor cells. Also, NK cells "help" in the activation of DCs by increasing their ability to produce pro-inflammatory cytokines and to stimulate lymphocyte T-helper ( $\mathrm{Th}_{1}$ ) and T-cytotoxic (Tc) cells against the tumor cells [31]. Barbarin et al. [90] provide new insights into the potential role of innate-CD8 ${ }^{+} \mathrm{T}$ cells (NK-like $\mathrm{CD} 8^{+}$cells) in physiopathological cancer progression. Based on data obtained in chronic myeloid leukemia, a myeloproliferative syndrome controlled by the immune system, and in solid tumors, they observe both the possible contribution of innate-CD8 ${ }^{+} \mathrm{T}$ cells to cancer disease control and their susceptibility to tumor immune subversion. During tumor progression, innate-CD8 ${ }^{+} \mathrm{T}$ lymphocytes are controlled by "immune checkpoints" [90]. It has also been shown that NK cells activated by DCs are up to 100 times more capable of producing the cytokine IL-12p70, which is resistant to inhibitory agents and can induce $\mathrm{a} \mathrm{Th}_{1}$ and Tc cell response [31]. These data have also been confirmed in clinical trials demonstrating the high efficacy of combination therapy with NK and DCs in skin melanoma [31]. Furthermore, it has been shown that TNF- $\alpha$ and IFN- $\gamma$ secreted by NK cells can activate Tc and NK cells [91] and inhibit tumor cell proliferation and angiogenesis [92-94]. However, it should be noted that IFN- $\gamma$ can also have adverse effects, because IFN- $\gamma$ released by ILC-1 cells at the early stage of the immune response may lead to tumor progression [95]. It has been reported that the presence of IFN- $\gamma$ adversely affects the in vitro culture of human melanoma cells, resulting in an aggressive phenotype and tumor growth [96]. Carrega et al. [43] describe the difference of NK cell distribution in cancer tissues. Neoplastic and corresponding healthy tissues from an array of human organs such as colorectal, lung, stomach, breast, adrenal gland and kidney were comparatively analyzed for the presence of NK cells. Statistically significant differences were detectable only in the breast and lung cancer group, where cancer tissues were enriched in CD56 ${ }^{\text {bright }}$ perforinlow noncytotoxic NK cells, compared to the healthy tissues sample [43]. Data presented by Simoni et al. [33] showed that the frequency of NK cells is higher in human colorectal tumor (73\% of cells) as compared to non-pathological colon tissues (34\% of cells), but there were no significant differences in the frequency of intra-epithelial-ILC1-like cells [33]. In lung cancer diseases, they noted that the number of NK cells is lower in human lung tumor ( $80 \%$ cells) as compared to non-pathological lung tissues (95\% cells), but there was a higher number of intra-epithelial-ILC1-like cells in human lung tumor ( $8 \%$ cells) than in non-pathological lung tissues (1\% cells) [33].

The role of ILC- 2 cells in antitumor protection is provided by the secretion of IL-13 and amphiregulin, which can inhibit type 1 responses correlated with Th1 lymphocytes. ILC-2 cells can also release a large amount of IL-5 which is necessary for the selective expansion of eosinophils [78]. The presence of eosinophilic granulocytes in the developing tumor area, i.e. colon cancer, esophagus, nasal cavity, larynx, pulmonary adenocarcinoma, bladder 
and prostate, indicates a better prognosis of patients [97-99]. In addition, IL-5 produced by mice ILC- 2 cells inhibited the metastasis of lung cancer and reduced tumor progression as a result of eosinophilia [98]. In addition, IL-5 produced by mice ILC- 2 cells inhibited the metastasis of lung cancer and reduced tumor progression as a result of eosinophilia [98]. It has also been demonstrated that IL-13, produced by IL-2 cells, is required to activate MDSC cells and can block the anti-tumor activity by transforming growth factor- $\beta$ (TGF- $\beta$ ) that is produced by MDSC cells, which controls the proliferation and differentiation of most cell types. Both TGF- $\beta$ and IL-13 can activate M2 macrophages to produce proangiogenic factors, such as vascular endothelial growth factor (VEGF), matrix metallopeptidase (MMP-9), and the growth factors epidermal growth factor (EGF) and fibroblast growth factor 2 (FGF-2), promoting the progression and metastasis of cancer cells [100-102]. It can therefore be stated that the role of ILC-2 cells in tumor growth is not only positive, because these cells can act in a pro-tumorigenic fashion by producing type 2 cytokines, including IL-13, which stimulates the microenvironment of the growing tumor and promotes metastasis. According to Simoni et al., the number of ILC-2 cells is similar in human colorectal tumor ( $1 \%$ cells) and in non-pathological colon tissues ( $3 \%$ cells), as well as in human lung tumor ( $3 \%$ cells) and non-pathological lung tissues ( $2 \%$ cells) [33].

The role of ILC-3 cells in cancer formation was found because these cells can cause chronic inflammation, i.e. inflammatory bowel disease (IBD) through IL-17, IL-22 or IL-23 secretion [103], and IBD increases the risk of colorectal cancer [104]. IL-22 produced by ILC-3 cells may be responsible for promoting colorectal cancer [11, 12]. IL-23 also activates ILC-3 cells to induce tumor growth in the intestines, ovaries, lung, breast, stomach, skin, liver and other organs [105-108]. High expression of IL-23 and release of IL-17 are unfavorable prognostic factors in cancer, because IL-17, by the activation of proangiogenic factor (VEGF), stimulates the formation of new blood vessels that supply the tumor [107]. In addition, IL-22 produced by ILC- 3 cells increases the proliferation of intestinal cancer cells by activating STAT2 pathways, and may cause long-term and recurrent inflammatory conditions that can predispose to cancer [109]. It has been reported that ILC-3 cells may possess natural cytotoxic receptors (NCR), such as NKp46, NKp30, and NKp44, which are characteristic for NK cells, and thus are capable of recognizing tumor cells or cells infected with virus [110-112]. It has been shown that ILC-3 cells, by activation of the NKp44 receptor and cytokine production, can penetrate the growing tumor, thereby affect- ing both tumor cells and fibroblasts presented in the tumor microenvironment. It was observed in patients with lung cancer that only a group of ILC-3 cells located in the immediate vicinity of the tumor has an active NKp44 receptor which was inactive in healthy lung tissue [113]. Reconstruction of ILC-3 cell populations in patients with acute myeloid leukemia (AML) have already been observed after 12 weeks of hematopoietic stem cell transplantation (HSCT) [114]. In the case of allogeneic HSCT transplants, patients may experience tissue damage and graft-versus-host disease (GvHD), which can cause life-threatening clinical symptoms such as inflammation of the skin and intestinal mucosa. It has also been demonstrated that IL-22, which is synthesized by ILC-3 cells, is a key regulator of tissue sensitivity to GvHD and protects intestinal stem cells from damage [114]. To confirm this, AML patients were examined and a correlation was observed between the presence of a large circulating population of ${\mathrm{NKp} 44^{+}} \mathrm{ILC}-3$ cells and the absence of GvHD disease symptoms [114]. According to Simoni et al., the frequency of NKp44--ILC3 cells is lower in human colorectal tumor (3\% cells) compared to non-pathological colon tissues (15\% cells), as well as NKp44+ILC3 cells (1\% and $23 \%$, respectively) [33]. In lung cancer diseases, the number of NKp44ILC3 cells is higher in human lung tumor ( $8 \%$ cells) as compared to non-pathological lung tissues ( $2 \%$ cells), but there were no significant differences in the number of NKp44+ILC3 cells, which are highly represented (more than $14 \%$ of total ILCS) in barrier tissues, such as tonsil, adenoid, colon and skin [33]. According to Huber et al. [115], ILC-3 cells producing IL-22 may also act protectively by limiting tissue damage in chronic colitis [115]. In turn, according to Kryczek et al. [116], IL-17 synthesized by ILC-3 can block the growth of tumor cells by the activation of $T$ cell-dependent mechanisms, which limits the process of colon cancer metastasis and colon carcinogenesis in mice. These data, as suggested by Carrega et al. [117], show that a better understanding of ILC cell biology, including cancer development, may provide new therapeutic pathways based on the activation and/ or blocking of receptors and intracellular ILC cell signaling, blocking the progression of cancer [117].

\section{Innate lymphoid cells and metabolic disorders}

It has been shown that ILC families 1,2 and 3 are involved in the formation of adipose tissue [118], are important components for the maintenance of metabolic homeostasis [2, 5-9], and are abundant in the liver of adults and in the fetal liver [38]. Different ILC subpopulations might exhibit different roles in some liver diseases. These seemingly 
paradoxical effects might depend on the inflammation state, as well as the tissue microenvironment. The protective or pathogenic roles of ILCS in liver diseases were summarized by Liu et al. [119]. The hepatic ILCS are involved in the immune regulation of viral hepatitis, mechanical liver injury and fibrosis. In obese people, adipocytes produce more IL-12 that can stimulate ILC-1 proliferation and accumulation in adipose tissue, and all proinflammatory cytokines produced by ILC-1 interfere with the immune response [120]. Moreover, ILC-1 cells can synthesize IFN- $\gamma$ and interact to polarize macrophages and promote insulin resistance in obesity [120]. It has been shown that both IFN- $\gamma$ and TNF- $\alpha$ synthesized by ILC- 1 are key factors in maintaining homeostasis in the body as they affect macrophages in adipose tissue [121] - tissue which is rich in NK cells, eosinophils, alternatively activated macrophages (AAMs) and ILC- 2 cells [8, 122, 123].

ILC-2 cells are responsible for regulating the amount of adipose tissue and influence the conversion of brown tissue into white fat tissue $[6,7$, $122,124,125]$. In addition, ILC-2 cells, by synthesizing IL-25 and IL-33, increase the number of AAM cells and eosinophilia in the fat tissue [7, 8] where IL-5 is synthesized $[124,125]$. Importantly, IL-5 deficiency may result in obesity and disrupt glucose metabolism [122]. It should be added that, in response to cold stress, adipocyte precursor cells of bone marrow origin can differentiate into brown adipose tissue cells with the help of eosinophils, AAM cells and catecholamine [6]. Additionally, cold stress can activate ILC- 2 cells by mobilizing eosinophils to produce IL-5 [6], and ILC-2 can directly induce the emergence of brown fat via the production of protein methionine/enkephalin, which increases the expression of thermogenin - a protein present in the mitochondria of brown adipose tissue [2].

Regarding the role of ILC-3 cells in metabolism, it has been shown that they respond to certain substances such as vitamin A and D [126-129]. It has been shown that retinoic acid, a biologically active metabolite of vitamin $A$, increases the production of IL-22 in ILC-3 cells by binding the retinoic acid receptor at locus 1122 [126]. This proves that vitamin A influences the function, distribution, and development of ILC-3 cells in the body. A deficiency of vitamin A can result in an increased number and survival time of ILC-2 cells, also by increasing the expression of $\mathrm{IL}-7 \mathrm{a}$ receptors [127]. It has also been shown that vitamin A deficiency decreases the number of ILC-3 in the intestines of adult mice [129], but vitamin $D$ receptor-knockout mice infected with Citrobacter rodentium have an increased number of ILC-3 cells in the intestine. This indicates that vitamin $D$ may have an inhibitory effect on these cells [128]. ILC-3 also regulates metabolic homeostasis via lymphotoxin $\beta$ (LT $\beta)$ and IL-22, which alleviates metabolic disorders and controls liver metabolism [9]. Studies have shown that diet- or genetic mutation-induced obese mice (mice ${ }^{\mathrm{db} / \mathrm{db}}$ ), when supplied with exogenous IL-22, exhibit decreased levels of triglycerides and cholesterol in serum, which lowers blood glucose and increases insulin sensitivity [9]. In addition, mice lacking lymphotoxin $\beta$ receptors ( $\mathrm{LT} \beta \mathrm{R}^{-/-}$were characterized by a lower weight compared to control mice with an active LT $\beta$ receptor, even after consuming a high fat diet [77]. It has been reported that reduced expression of IL-22 causes a decrease in the expression of the antibacterial peptide RegIIIc, which may lead to the expansion of Candidatus savagella bacterium [130]. ILC-3 cells, as a valuable source of the IL-22 cytokine required for weight gain, are important regulators of metabolic homeostasis, play a role in maintaining the integrity of the body's barriers, and are involved in the production of anti-microbial proteins [77].

\section{Innate lymphoid cells and inflammation}

Under inflammatory conditions, according to the results of Simoni et al. [33], more than 95\% of ILCS recruited were composed of NK or intraepithelial-ILC1-like cells in all nine different healthy tissues and three pathological tissues analyzed by them. In contrast to non-mucosal and lung tissues, oral and gastrointestinal mucosal and skin tissues contained a high frequency of helper-type ILCS, which is consistent with the role of these cells in human barrier surface immunity and which has already been partially confirmed in mice [13]. The role and contribution of ILC cells in inflammatory and allergic reactions, i.e. asthma, atopic dermatitis and chronic sinusitis, has been confirmed in principle for the ILC-2 and ILC-3 cells [131-133]. It has been documented that genes of atopic diseases, e.g. genes encoding IL-33 and its receptor, as well as genes encoding TSLP, IL-4, IL-5 and IL-13, are key factors for ILC-2 cell activation [134]. It has been reported that papain, which induces asthma in $\mathrm{Rag}^{-/-}$mice, does not induce asthma in $\mathrm{Rag}^{-1-} \| 12 \mathrm{rg}^{-1-}$ double knockout mice or $\mathrm{Rag}^{-/}$mice with a low number of ILC-2 cells [135]. Resende et al. [136] identified a novel population of nonconventional IFN- $\gamma$-producing cells characterized by expression of Thy 1.2 and a lack of lymphoid, myeloid and NK lineage markers. Their results demonstrate that a population of Thy $1.2^{+}$non-NK innate-like cells present in the liver expresses IFN- $\gamma$ and can confer protection against Mycobacterium avium infection in immunocompromised mice lacking both the Rag2 and the common $\gamma$-chain $(\gamma \mathrm{c})$ genes $\left(\mathrm{Rag}^{-/} \gamma \mathrm{C}^{-/}\right)$, indicating their innate nature and their $\gamma c$ cytokine independence [136]. 
The role of ILC-2 cells has also been demonstrated in lung [135] and skin [137] inflammation, which depends on IL-25, IL-33, and the IL-4 cytokine derived from basophils. It has been shown that ILC-2 cells cause excessive respiratory tract reactivity and can predispose to allergic alveolitis, which in turn may lead to the development of interstitial lung disease [135]. In addition, by secreting the cytokines IL-5 and IL-13, these cells can activate eosinophils, which are extremely important cells in allergies $[125,138]$. By producing IL-9, ILC-2 cells can cause allergic inflammation of the respiratory tracts [139]. In asthmatic patients, a large amount of circulating ILC-2 was found, but mouse studies [140] showed that corticosteroid treatment resulted in a decreased number of ILC-2 cells after IL-33 activation, and this cytokine is intensively produced during viral infections and the allergic state by epithelial cells and/or lung follicular macrophages [141]. The role of ILC-2 has also been demonstrated in mice during skin inflammation, during supplementation with calcipotriol (analogue of vitamin D), during supplementation with cytokine IL-2, and in response to the over-expression of IL-33 [141].

The role of ILC-3 cells in inflammatory processes has been demonstrated in a mouse model of dermatitis which found increased numbers of ILC-3 cells as a result of IL-33 overexpression [142]. A high level of ILC-3 cells has also been reported in human gastrointestinal allergies, e.g. enteritis [143-145], and induced inflammation of the small and large intestine, caused by Helicobacter hepaticus, $H$. typhlonus, and Salmonella typhimurium [146]. In Crohn's disease (CD), we can observe a decreased population of ILC-3 and, surprisingly, an increased population of ILC-1, which seems to come from the population of ILC-3 cells [147]. Crohn's disease is also characterized by the increased production of IL-17 cytokines by ILC-3 cells [144]. It is believed that the role of ILC-3 is associated with allergic diseases and depends on the diet, the influence of the external environment, and the functioning of the immune system [148]. ILC-3 cells were found during inflammatory responses in GVHD transplant recipients, and it has also been reported that ILC-3 cells circulating in the blood, pre- and post- transplant, can reduce the risk of GVHD $[10,75]$. Hanash et al. documented that in the bone marrow of transplanted mice, ILC-3 cells can produce IL-22, which is a cytokine that protects bone marrow stem cells from damage [10].

\section{Conclusions}

Innate lymphoid cells are a multifunctional group of immune cells. Their ability to rapidly secrete immunoregulatory cytokines allows them to con- tribute to early immune responses after infection. Taking a closer look at the role of ILC cells, mainly in the field of immunity, will create conditions for the development of new procedures that may lead to improved health care. The contribution of ILC cells as the "guardians" in the homeostasis and immunity of mammals, including humans, is constantly examined. A study published by the agency Immunological Genome Consortium [37] described the transcriptional expression profiles of the ILC family in various tissues and defined the code of ILC. They also gave a suggestion about the new potential functions of ILCs for protecting health, i.e. as a source of diagnostic markers and cells, whose presence can be used in diagnostics, as well as in basic research. Studies analyzing all known ILC subsets for individual tissues in parallel are lacking and the differences in murine and human immunology pose a challenge. Simoni et al. [33] were the first group to extensively analyze the phenotypic characteristics of human ILCs. By using mass cytometry (CyTOF), they simultaneously analyzed 29 parameters in multiple primary, healthy and pathological human samples. These results provide a global, comprehensive and detailed description of ILC populations and their heterogeneity across individuals and tissues. All presented data give a hope for further study of the ILC population in normal and abnormal conditions.

\section{Acknowledgments}

We would like to thank Zachariah Payne Sellers for his language and translation support.

\section{Conflict of interest}

The authors declare no conflict of interest.

\section{References}

1. McKenzie AN, Spits H, Eberl G. Innate lymphoid cells in inflammation and immunity. Immunity 2014; 41: 366-74.

2. Brestoff JR, Kim BS, Saenz SA, et al. Group 2 innate lymphoid cells promote beiging of white adipose tissue and limit obesity. Nature 2015; 519: 242-6.

3. Hepworth MR, Monticelli LA, Fung TC, et al. Innate lymphoid cells regulate $C D 4$ ? T-cell responses to intestinal commensal bacteria. Nature 2013; 498: 113-7.

4. Hepworth MR, Fung TC, Masur SH, et al. Immune tolerance. Group 3 innate lymphoid cells mediate intestinal selection of commensal bacteria-specific $\mathrm{CD}^{+}{ }^{+} \mathrm{T}$ cells. Science 2015; 348: 1031-5.

5. Lee MW, Odegaard JI, Mukundan L, et al. Activated type 2 innate lymphoid cells regulate beige fat biogenesis. Cell 2015; 160: 74-87.

6. Qiu Y, Nguyen KD, Odegaard JI, et al. Eosinophils and type 2 cytokine signaling in macrophages orchestrate development of functional beige fat. Cell 2014; 157 : 1292-1308.

7. Molofsky AB, Nussbaum JC, Liang HE, et al. Innate lymphoid type 2 cells sustain visceral adipose tissue eosin- 
ophils and alternatively activated macrophages. J Exp Med 2013; 210: 535-49.

8. Hams E, Locksley RM, McKenzie AN, Fallon PG. Cutting edge: IL-25 elicits innate lymphoid type 2 and type II NKT cells that regulate obesity in mice. J Immunol 2013; 191: 5349-53.

9. Wang X, Ota N, Manzanillo P, et al. Interleukin-22 alleviates metabolic disorders and restores mucosal immunity in diabetes. Nature 2004; 514: 237-41.

10. Hanash AM, Dudakov JA, Hua G, et al. Interleukin-22 protects intestinal stem cells from immune-mediated tissue damage and regulates sensitivity to graft versus host disease. Immunity 2012; 37: 339-50.

11. Boulard O, Kirchberger S, Royston DJ, Maloy KJ, Powrie FM. Identification of a genetic locus controlling bacteriadriven colitis and associated cancer through effects on innate inflammation. J Exp Med 2012; 209: 1309-24.

12. Kirchberger S, Royston DJ, Boulard O, et al. Innate lymphoid cells sustain colon cancer through production of interleukin-22 in a mouse model. J Exp Med 2013; 210: 917-31.

13. Artis D, Spits $H$. The biology of innate lymphoid cells. Nature 2015; 517: 293-301.

14. Hazenberg MD, Spits $H$. Human innate lymphoid cells. Blood 2014; 124: 700-9.

15. Spits H, Artis D, Colonna M, et al. Innate lymphoid cells a proposal for uniform nomenclature. Nat Rev Immunol 2013; 13: 145-9.

16. Cortez VS, Robinette ML, Colonna M. Innate lymphoid cells: new insights into function and development. Curr Opin Immunol 2015; 32: 71-7.

17. von Moltke J, Locksley RM. I-L-C-2 it: type 2 immunity and group 2 innate lymphoid cells in homeostasis. Curr Opin Immunol 2014; 31: 58-65.

18. Peterson LW, Artis D. Intestinal epithelial cells: regulators of barrier function and immune homeostasis. Nat Rev Immunol 2014; 14: 141-53.

19. Klose CS, Diefenbach A. Transcription factors controlling innate lymphoid cell fate decisions. Curr Top Microbiol Immunol 2014; 381: 215-55.

20. Gasteiger G, Rudensky AY. Interactions between innate and adaptive lymphocytes. Nat Rev Immunol 2014; 14: 631-9.

21. Diefenbach A, Colonna M, Koyasu S. Development, differentiation, and diversity of innate lymphoid cells. Immunity 2014; 41: 354-65.

22. Bordon Y. Innate lymphoid cells: on the origin of ILCS. Nat Rev Immunol 2014; 14: 133.

23. Walker JA, Barlow JL, McKenzie AN. Innate lymphoid cells - how did we miss them? Nat Rev Immunol 2013; 13: 75-87.

24. Philip NH, Artis D. New friendships and old feuds: relationships between innate lymphoid cells and microbia communities. Immunol Cell Biol 2013; 91: 225-31.

25. Spits H, Cupedo T. Innate lymphoid cells: emerging insights in development, lineage relationships, and function. Annu Rev Immunol 2012; 30: 647-75.

26. Sonnenberg GF, Artis D. Innate lymphoid cell interactions with microbiota: implications for intestinal health and disease. Immunity 2012; 37: 601-10.

27. Rankin L, Groom J, Mielke LA, Seillet C, Belz GT. Diversity, function, and transcriptional regulation of gut innate lymphocytes. Front Immunol 2013; 4: 22.

28. Adamiak M, Tokarz-Deptuła B, Deptuła W. Charakterystyka naturalnych komórek limfoidalnych (ILC). Postepy Hig Med Dosw (Online) 2014; 68: 1464-71.
29. Mjösberg J, Mazzurana L. ILC-poiesis: making tissue ILCs from blood. Immunity 2017; 46: 344-6.

30. Fang D, Zhu J. Dynamic balance between master transcription factors determines the fates and functions of CD4 T cell and innate lymphoid cell subsets. J Exp Med 2017; 214: 1861-76.

31. Kalinski P, Mailliard RB, Giermasz A, et al. Natural killerdendritic cell cross-talk in cancer immunotherapy. Expert Opin Biol Ther 2005; 5: 1303-15.

32. Hazenberg MD, Spits H. Human innate lymphoid cells. Blood 2014; 124: 700-9.

33. Simoni $Y$, Fehlings $M$, Kløverpris $H N$, et al. Human innate lymphoid cell subsets possess tissue-type based heterogeneity in phenotype and frequency. Immunity 2017; 46: 148-61.

34. Xu H, Wang X, Liu DX, Moroney-Rasmussen T, Lackner AA, Veazey RS. IL-17-producing innate lymphoid cells are restricted to mucosal tissues and are depleted in SIV-infected macaques. Mucosal Immunol 2012; 5: 658-69.

35. Bal SM, Bernink JH, Nagasawa M, et al. IL-1 $\beta$, IL-4 and IL-12 control the fate of group 2 innate lymphoid cells in human airway inflammation in the lungs. Nat Immunol 2016; 17: 636-45.

36. Silver JS, Kearley J, Copenhaver AM, et al. Inflammatory triggers associated with exacerbations of COPD orchestrate plasticity of group 2 innate lymphoid cells in the lungs. Nat Immunol 2016; 17: 626-35.

37. Robinette ML, Fuchs A, Cortez VS, et al. Immunological Genome C. Transcriptional programs define molecular characteristics of innate lymphoid cell classes and subsets. Nat Immunol 2015; 16: 306-17.

38. Klose CS, Flach M, Mohle L, et al. Differentiation of type 1 ILCs from a common progenitor to all helper-like innate lymphoid cell lineages. Cell 2014; 157: 340-56.

39. Fuchs A, Vermi W, Lee JS, et al. Intraepithelial type 1 innate lymphoid cells are a unique subset of IL-12- and IL-15-responsive IFN-gamma-producing cells. Immunity 2013; 38: 769-81.

40. Bernink JH, Peters CP, Munneke M, et al. Human type 1 innate lymphoid cells accumulate in inflamed mucosal tissues. Nat Immunol 2013; 14: 221-9.

41. Sun JC, Lanier LL. NK cell development, homeostasis and function: parallels with CD8? T cells. Nat Rev Immunol 2011; 11: 645-57.

42. Maloy KJ, Uhlig HH. ILC1 populations join the border patrol. Immunity 2013; 38: 630-2.

43. Carrega P, Bonaccorsi I, Di Carlo E, et al. CD56(bright) perforin(low) noncytotoxic human NK cells are abundant in both healthy and neoplastic solid tissues and recirculate to secondary lymphoid organs via afferent lymph. J Immunol 2014; 192: 3805-15.

44. Eken A, Donmez-Altuntas H. Innate Lymphoid Cells (Non-NK ILCs). Lymphocyte Updates - Cancer, Autoimmunity and Infection. InTech, London 2017, Chapter 7.

45. Mavropoulos A, Sully G, Cope AP, Clark AR. Stabilization of IFN-gamma mRNA by MAPK p38 in IL-12- and IL-18-stimulated human NK cells. Blood 2005; 105: 282-8.

46. Wang S, Xia P, Chen Y, et al. Natural killer-like B cells prime innate lymphocytes against microbial infection. Immunity 2016; 45: 131-44.

47. Yang Q, Saenz SA, Zlotoff DA, Artis D, Bhandoola A. Cutting edge: natural helper cells derive from lymphoid progenitors. J Immunol 2011; 187: 5505-9.

48. Chang YJ, Kim HY, Albacker LA, et al. Innate lymphoid cells mediate influenza-induced airway hyper-reactivity independently of adaptive immunity. Nat Immunol 2011; 12: 631-8. 
49. Mjosberg JM, Trifari S, Crellin NK, et al. Human IL-25and IL-33-responsive type 2 innate lymphoid cells are defined by expression of CRTH2 and CD161. Nat Immunol 2011; 12: 1055-62.

50. Eiwegger T, Akdis CA. IL-33 links tissue cells, dendritic cells and Th2 cell development in a mouse model of asthma. Eur J Immunol 2011; 41: 1535-8.

51. Halim TY, MacLaren A, Romanish MT, Gold MJ, McNagny KM, Takei F. Retinoic-acid-receptor-related orphan nuclear receptor alpha is required for natural helper cell development and allergic inflammation. Immunity 2012; 37: 463-74.

52. Price AE, Liang HE, Sullivan BM, et al. Systemically dispersed innate IL-13-expressing cells in type 2 immunity. Proc Natl Acad Sci USA 2010; 107: 11489-94.

53. Mjösberg J, Bernink J, Golebski K, et al. The transcription factor GATA3 is essential for the function of human type 2 innate lymphoid cells. Immunity 2012; 37: 649-59.

54. Moro K, Yamada T, Tanabe M, et al. Innate production of $\mathrm{T}(\mathrm{H}) 2$ cytokines by adipose tissue-associated c-Kit(+) Sca-1(+) lymphoid cells. Nature 2010; 463: 540-4.

55. Neill DR, Wong SH, Bellosi A, et al. Nuocytes represent a new innate effector leukocyte that mediates type- 2 immunity. Nature 2010; 464: 1367-70.

56. Wong SH, Walker JA, Jolin HE, et al. Transcription factor RORalpha is critical for nuocyte development. Nat Immunol 2012; 13: 229-36.

57. Roediger B, Weninger W. Group 2 innate lymphoid cells in the regulation of immune responses. Adv Immunol 2015; 125: 111-54.

58. Roediger B, Kyle R, Yip KH, et al. Cutaneous immunosurveillance and regulation of inflammation by group 2 innate lymphoid cells. Nat Immunol 2013; 14: 564-73.

59. Ho J, Bailey M, Zaunders J, et al. Group 2 innate lymphoid cells (ILC2s) are increased in chronic rhinosinusitis with nasal polyps or eosinophilia. Clin Exp Allergy 2015; 45: 394-403.

60. Miljkovic D, Bassiouni A, Cooksley C, et al. Association between group 2 innate lymphoid cells enrichment, nasal polyps and allergy in chronic rhinosinusitis. Allergy 2014; 69: 1154-61.

61. Fallon PG, Ballantyne SJ, Mangan NE, et al. Identification of an interleukin (IL)-25-dependent cell population that provides IL-4, IL-5, and IL-13 at the onset of helminth expulsion. J Exp Med 2006; 203: 1105-16.

62. Doherty TA, Khorram N, Lund S, Mehta AK, Croft M, Broide DH. Lung type 2 innate lymphoid cells express cysteinyl leukotriene receptor 1 , which regulates Th2 cytokine production. J Allergy Clin Immunol 2013; 132: 205-13.

63. Turner JE, Morrison PJ, Wilhelm C, et al. IL-9-mediated survival of type 2 innate lymphoid cells promotes damage control in helminth-induced lung inflammation. J Exp Med 2013; 210: 2951-65.

64. Monticelli LA, Osborne LC, Noti M, Tran SV, Zaiss DM, Artis D. IL-33 promotes an innate immune pathway of intestinal tissue protection dependent on amphiregulin-EGFR interactions. Proc Natl Acad Sci USA 2015; 112: 10762-7.

65. Yang Q, Monticelli LA, Saenz SA, et al. T cell factor 1 is required for group 2 innate lymphoid cell generation. Immunity 2013; 38: 694-704.

66. Kim BS, Siracusa MC, Saenz SA, et al. TSLP elicits IL-33independent innate lymphoid cell responses to promote skin inflammation. Sci Transl Med 2013; 5: 170ra16.

67. Salimi M, Barlow IL, Saunders SP, et al. A role for IL-25 and IL-33-driven type-2 innate lymphoid cells in atopic dermatitis. J Exp Med 2013; 210: 2939-50.
68. Satoh-Takayama N, Vosshenrich CA, Lesjean-Pottier S, et al. Microbial flora drives interleukin 22 production in intestinal NKp46? cells that provide innate mucosal immune defense. Immunity 2008; 29: 958-70.

69. Montaldo E, Juelke K, Romagnani C. Group 3 innate lym phoid cells (ILC3s): origin, differentiation, and plasticity in humans and mice. Eur J Immunol 2015; 45: 2171-82.

70. Takatori H, Kanno Y, Watford WT, et al. Lymphoid tissue inducer-like cells are an innate source of IL-17 and IL-22. J Exp Med 2009; 206: 35-41.

71. Cella M, Fuchs A, Vermi W, et al. A human natural killer cell subset provides an innate source of IL-22 for mucosal immunity. Nature 2009; 457: 722-5.

72. Mortha A, Chudnovskiy A, Hashimoto D, et al. Microbiota-dependent crosstalk between macrophages and ILC3 promotes intestinal homeostasis. Science 2014; 343 1249288.

73. Sonnenberg GF, Monticelli LA, Alenghat T, et al. Innate lymphoid cells promote anatomical containment of lymphoidresident commensal bacteria. Science 2012; 336 1321-5.

74. Sonnenberg GF, Monticelli LA, Elloso MM, Fouser LA Artis D. CD4(+) lymphoid tissue-inducer cells promote innate immunity in the gut. Immunity 2011; 34: 122-34.

75. Munneke JM, Bjorklund AT, Mjosberg JM, et al. Activated innate lymphoid cells are associated with a reduced susceptibility to graft versus host disease. Blood 2014; 124: 812-21.

76. Luci C, Reynders A, Ivanov II, et al. Influence of the transcription factor RORgammat on the development of NKp46+ cell populations in gut and skin. Nat Immunol 2009; 10: 75-82.

77. Scandella E, Bolinger B, Lattmann E, et al. Restoration of lymphoid organ integrity through the interaction of lymphoid tissue-inducer cells with stroma of the T cell zone. Nat Immunol 2008; 9: 667-75.

78. Vacca P, Montaldo E, Croxatto D, et al. Identification of diverse innate lymphoid cells in human decidua. Mucosal Immunol 2015; 8: 254-64.

79. Cupedo T, Crellin NK, Papazian N, et al. Human fetal lymphoid tissue-inducer cells are interleukin 17-producing precursors to RORC+CD127+ natural killer-like cells. Nat Immunol 2009; 10: 66-74.

80. Crellin NK, Trifari S, Kaplan CD, Satoh-Takayama N, Di Santo JP, Spits H. Regulation of cytokine secretion in human CD127(+) LTi-like innate lymphoid cells by Toll-like receptor 2 . Immunity 2010; 33: 752-64.

81. Ungefroren H, Sebens S, Seidl D, Lehnert H, Hass R. Interaction of tumor cells with the environment. Cell Comm Signal 2011; 9: 18-24.

82. Zammaron BF, Chen WJ. Dual roles of immune cells and their factors in cancer development and progression. Int J Biol Sci 2011; 7: 651-9.

83. Zhang W, Huang P. Cancer-stromal interactions. Cancer Biol Ther 2011; 11: 150-6.

84. Fan F, Schimming A, Jaeger D, Podar K. Targeting the tumor microenvironment: focus on angiogenesis. J Oncol 2012; 2012: 2811261

85. Ostrand-Rosenberg S, Sinha P. Myeloid-derived supressor cells: linking inflammation and cancer. J Immuno 2009; 182: 4499-506.

86. Spits H, Cupedo T. Innate lymphoid cells: emerging insights in development, lineage relationships, and function. Annu Rev Immunol 2012; 30: 647-75.

87. Nowarski R, Gagliani N, Huber S, Flavell RA. Innate immune cells in inflammation and cancer. Cancer Immunol Res 2013; 1: 77-84. 
88. Zitvogel L, Kepp O, Galluzzi L, Kroemer G. Inflammasomes in carcinogenesis and anticancer immune responses. Nat Immunol 2012; 13: 343-51.

89. Levy EM, Robert MP, Mordoh J. Natural killer cells in human cancer: from biological functions to clinical applications. J Biomed Biotech 2011; 2011: 676198.

90. Barbarin A, Cayssials E, Jacomet F, et al. Phenotype of NK-like CD8(+) T cells with innate features in humans and their relevance in cancer diseases. Front Immunol 2017; 8: 316.

91. Terabe M, Berzovsky J. The role of NKT in tumor immunity. Adv Cancer Res 2008; 101: 277-348.

92. Rüegg C, Yilmaz A, Bieler G, Bamat J, Chaubert P, Lejeune FJ. Evidence for the involvement of endothelial cell integrin alphaVbeta3 in the disruption of the tumor vasculature induced by TNF and IFN-gamma. Nat Med 1998; 4: 408-14.

93. Coughlin CM, Salhany KE, Gee MS, et al. Tumor cell responses to IFNgamma affect tumorigenicity and response to IL-12 therapy and antiangiogenesis. Immunity 1998; 9: 25-34.

94. Beatty G, Paterson Y. IFN-gamma-dependent inhibition of tumor angiogenesis by tumor-infiltrating CD4+ $T$ cells requires tumor responsiveness to IFN-gamma. J Immunol 2001; 166: 2276-82.

95. Zaidi MR, Merlino G. The two faces of interferon-gamma in cancer. Clin Cancer Res 2011; 17: 6118-24.

96. Garbe C, Krasagakis K, Zouboulis CC, et al. Antitumor activities of interferon alpha, beta, and gamma and their combinations on human melanoma cells in vitro: chang es of proliferation, melanin synthesis, and immunophenotype. J Invest Dermatol 1990; 95 (6 Suppl): 231-7.

97. Pretlow TP, Keith EF, Cryar AK, et al. Eosinophil infiltration of human colonic carcinomas as a prognostic indicator. Cancer Res 1983; 43: 2997-3000.

98. Costello R, O'Callaghan T, Sebahoun G. Eosinophils and antitumour response. Rev Med Interne 2005; 26: 47984.

99. Luna-More S, Florez P, Ayala A, Diaz F, Santos A. Neutral and acid mucins and eosinophil and argyrophil crystalloids in carcinoma and atypical adenomatous hyperplasia of the prostate. Pathol Res Pract 1997; 193: 291-8.

100. Coussens LM, Werb Z. Inflammation and cancer. Nature 2002; 420: 860-7.

101. Balkwill F, Charles KA, Mantovani A. Smoldering and polarized inflammation in the initiation and promotion of malignant disease. Cancer Cell 2005; 7: 211-7.

102. Qian BZ, Pollard JW. Macrophage diversity enhances tumor progression and metastasis. Cell 2010; 141: 39-51.

103. Geremia A, Arancibia-Cárcamo CV, Fleming MP, et al. IL-23-responsive innate lymphoid cells are increased in inflammatory bowel disease. J Exp Med 2011; 208: 1127-33.

104. Triantafillidis JK, Nasioulas G, Kosmidis PA. Colorectal cancer and inflammatory bowel disease: epidemiology, risk factors, mechanisms of carcinogenesis and prevention strategies. Anticancer Res 2009; 29: 2727-37.

105. Chan IH, Jain R, Tessmer MS, et al. Interleukin-23 is sufficient to induce rapid de novo gut tumorigenesis, independent of carcinogens, through activation of innate lymphoid cells. Mucosal Immunol 2014; 7: 842-56.

106. Langowski JL, Zhang X, Wu L, et al. IL-23 promotes tumour incidence and growth. Nature 2006; 442: 461-5.

107. Grivennikov SI, Wang K, Mucida D, et al. Adenomalinked barrier defects and microbial products drive IL-23/IL-17-mediated tumour growth. Nature 2012; 491: 254-8.
108. Li J, Lau G, Chen L, et al. Interleukin 23 promotes hepatocellular carcinoma metastasis via NF-kappa B induced matrix metalloproteinase 9 expression. PLoS One 2012; 7: e46264.

109. Pan B, Shen J, Cao J, et al. Interleukin-17 promotes angiogenesis by stimulating VEGF production of cancer cells via the STAT3/GIV signaling pathway in nonsmall-cell lung cancer. Sci Rep 2015; 5: 16053.

110. Sivori S, Pende D, Bottino C, et al. NKp46 is the major triggering receptor involved in the natural cytotoxicity of fresh or cultured human NK cells. Correlation between surface density of NKp46 and natural cytotoxicity against autologous, allogeneic or xenogeneic target cells. Eur J Immunol 1999; 29: 1656-66.

111. Pende D, Parolini S, Pessino A, et al. Identification and molecular characterization of NKp30, a novel triggering receptor involved in natural cytotoxicity mediated by human natural killer cells. J Exp Med 1999; 190: 1505-16.

112. Vitale M, Bottino C, Sivori S, et al. NKp44, a novel triggering surface molecule specifically expressed by activated natural killer cells, is involved in non-major histocompatibility complex-restricted tumor cell lysis. J Exp Med 1998; 187: 2065-72.

113. Carrega P, Loiacono F, Di Carlo E, et al. NCR(+)ILC3 concentrate in human lung cancer and associate with intratumoral lymphoid structures. Nat Commun 2015; 6: 8280.

114. Munneke JM, Bjorklund AT, Mjosberg JM, et al. Activated innate lymphoid cells are associated with a reduced susceptibility to graft-versus-host disease. Blood 2014; 124: 812-21.

115. Huber S, Gagliani N, Zenewicz LA, et al. IL-22BP is regulated by the inflammasome and modulates tumorigenesis in the intestine. Nature 2012; 491: 259-63.

116. Kryczek I, Wei S, Szeliga W, Vatan L, Zou W. Endogenous IL-17 contributes to reduced tumor growth and metastasis. Blood 2009; 114: 357-9.

117. Carrega P, Campana S, Bonaccorsi I, Ferlazzo G. The Yin and Yang of innate lymphoid cells in cancer. Immunol Lett 2016; 179: 29-35.

118. Narni-Mancinelli E, Vivier E. Killer ILCs in the fat. Immunity 2017; 46: 169-71.

119. Liu M, Zhang C. The role of innate lymphoid cells in immune-mediated liver diseases. Front Immunol 2017; 8: 695.

120. O'Sullivan TE, Rapp M, Fan X, et al. Adipose-resident group 1 innate lymphoid cells promote obesity-associated insulin resistance. Immunity 2016; 45: 428-41.

121. Chawla A, Nguyen KD, Goh YP. Macrophage-mediated inflammation in metabolic disease. Nat Rev Immunol 2011; 11: 738-49.

122. Wu D, Molofsky AB, Liang HE, et al. Eosinophils sustain adipose alternatively activated macrophages associated with glucose homeostasis. Science 2010; 332: 243-7.

123. Bosurgi L, Bernink JH, Delgado Cuevas V, et al. Paradoxical role of the proto-oncogene $\mathrm{Axl}$ and Mer receptor tyrosine kinases in colon cancer. Proc Natl Acad Sci USA 2013; 110: 13091-6.

124. Yamaguchi Y, Hayashi Y, Sugama Y, et al. Highly purified murine interleukin 5 (IL-5) stimulates eosinophil function and prolongs in vitro survival. IL-5 as an eosinophil chemotactic factor. J Exp Med 1988; 167: 1737-42.

125. Nussbaum JC, Van Dyken SJ, von Moltke J, et al. Type 2 innate lymphoid cells control eosinophil homeostasis. Nature 2013; 502: 245-8.

126. Mielke LA, Jones SA, Raverdeau M, et al. Retinoic acid expression associates with enhanced IL-22 produc- 
tion by gammadelta $T$ cells and innate lymphoid cells and attenuation of intestinal inflammation. J Exp Med 2013; 210: 1117-24.

127. Spencer SP, Wilhelm C, Yang Q, et al. Adaptation of innate lymphoid cells to a micronutrient deficiency promotes type 2 barrier immunity. Science 2014; 343: 432-7.

128. Chen J, Waddell A, Lin YD, Cantorna MT. Dysbiosis caused by vitamin $\mathrm{D}$ receptor deficiency confers colonization resistance to Citrobacter rodentium through modulation of innate lymphoid cells. Mucosal Immunol 2015; 8: 618-26.

129. van de Pavert SA, Ferreira M, Domingues RG, et al. Maternal retinoids control type 3 innate lymphoid cells and set the offspring immunity. Nature 2014; 508: 123-7.

130. Qiu J, Guo X, Chen ZM, et al. Group 3 innate lymphoid cells inhibit T-cellmediated intestinal inflammation through aryl hydrocarbon receptor signaling and regulation of microflora. Immunity 2013; 39: 386-99.

131. Kim BS, Siracusa MC, Saenz SA, et al. TSLP elicits IL-33-independent innate lymphoid cell responses to promote skin inflammation. Sci Transl Med 2013; 5: 170 ra16.

132. Halim TY, Krauss RH, Sun AC, Takei F. Lung natural helper cells are a critical source of Th2 cell-type cytokines in protease allergen-induced airway inflammation. Immunity 2012; 36: 451-63.

133. Imai Y, Yasuda K, Sakaguchi Y, et al. Skin-specific expression of IL-33 activates group 2 innate lymphoid cells and elicits atopic dermatitis-like inflammation in mice. Proc Natl Acad Sci USA 2013; 110: 13921-6.

134. Li J, Zhang Y, Zhang L. Discovering susceptibility genes for allergic rhinitis and allergy using a genome-wide association study strategy. Curr Opin Allergy Clin Immunol 2015; 15: 33-40.

135. Motomura Y, Morita H, Moro K, et al. Basophil-derived interleukin-4 controls the function of natural helper cells, a member of ILC2s, in lung inflammation. Immunity 2014; 40: 758-71.

136. Resende M, Cardoso MS, Ribeiro AR, et al. Innate IFN-gamma-producing cells developing in the absence of IL-2 receptor common gamma-chain. J Immunol 2017; 199: 1429-39.

137. Kim BS, Wang K, Siracusa MC, et al. Basophils promote innate lymphoid cell responses in inflamed skin. J Immunol 2014; 193: 3717-25.

138. Jung Y, Wen T, Mingler MK, et al. IL-1beta in eosinophil-mediated small intestinal homeostasis and IgA production. Mucosal Immunol 2015; 8: 930-42.

139. Wilhelm C, Hirota K, Stieglitz B, et al. An IL-9 fate reporter demonstrates the induction of an innate IL-9 response in lung inflammation. Nat Immunol 2011; 12: 1071-7.

140. Kabata H, Moro K, Fukunaga K, et al. Thymic stromal lymphopoietin induces corticosteroid resistance in natural helper cells during airway inflammation. Nat Commun 2013; 4: 2675.

141. Chang YJ, Kim HY, Albacker LA, et al. Innate lymphoid cells mediate influenza-induced airway hyper-reactivity independently of adaptive immunity. Nat Immunol 2011; 12: 631-8.

142. Imai Y, Yasuda K, Sakaguchi Y, et al. Skin-specific expression of IL-33 activates group 2 innate lymphoid cells and elicits atopic dermatitis-like inflammation in mice. Proc Natl Acad Sci USA 2013; 110: 13921-6.

143. Duerr RH, Taylor KD, Brant SR, et al. A genome-wide association study identifies IL23R as an inflammatory bowel disease gene. Science 2006; 314: 1461-3.
144. Geremia A, Arancibia-Cárcamo CV, Fleming MP, et al. IL-23-responsive innate lymphoid cells are increased in inflammatory bowel disease. J Exp Med 2011; 208: 1127-33.

145. Maloy KJ, Powrie F. Intestinal homeostasis and its breakdown in inflammatory bowel disease. Nature 2011; 474: 298-306.

146. Buonocore S, Ahern PP, Uhlig HH, et al. Innate lymphoid cells drive interleukin-23-dependent innate intestinal pathology. Nature 2010; 464: 1371-5.

147. Fuchs A, Vermi W, Lee JS, et al. Intraepithelial type 1 innate lymphoid cells are a unique subset of IL-12- and IL-15-responsive IFN-gamma-producing cells. Immunity 2013; 38: 769-81.

148. Hashiguchi M, Kashiwakura Y, Kojima H, Kobayashi A, Kanno Y, Kobata T. IL-33 activates eosinophils of visceral adipose tissue both directly and via innate lymphoid cells. Eur J Immunol 2015; 45: 876-85. 